\title{
BMJ open Independent association between low serum amylase and non-alcoholic fatty liver disease in asymptomatic adults: a cross-sectional observational study
}

\author{
Kei Nakajima, ${ }^{1,4}$ Haruki Oshida, ${ }^{1}$ Toshitaka Muneyuki, ${ }^{2}$ Masafumi Saito, ${ }^{1}$ \\ Yumiko Hori, ${ }^{1}$ Hiroshi Fuchigami, ${ }^{3}$ Masafumi Kakei, ${ }^{2}$ Hiromi Munakata ${ }^{4}$
}

To cite: Nakajima $\mathrm{K}$, Oshida $\mathrm{H}$, Muneyuki T, et al. Independent association between low serum amylase and non-alcoholic fatty liver disease in asymptomatic adults: a cross-sectional observational study. BMJ Open 2013;3:e002235. doi:10.1136/bmjopen-2012002235

- Prepublication history and additional material for this paper are available online. To view these files please visit the journal online (http://dx.doi.org/10.1136/ bmjopen-2012-002235).

Received 18 October 2012 Revised 11 December 2012 Accepted 11 December 2012

This final article is available for use under the terms of the Creative Commons Attribution Non-Commercial 2.0 Licence; see http://bmjopen.bmj.com

For numbered affiliations see end of article

Correspondence to Kei Nakajima; keinaka@josai.ac.jp

\section{ABSTRACT}

Objectives: Low serum amylase (LSA) was reported to be associated with obesity, metabolic syndrome (MetS) and diabetes. However, it is unknown as to whether LSA is associated with non-alcoholic fatty liver disease (NAFLD), a hepatic manifestation of MetS and insulin resistance. Therefore, we performed a clinical epidemiological study to investigate this potential association.

Design: A cross-sectional observational study with multivariate analysis.

Setting: Subjects were recruited in a healthcare centre in Saitama, an eastern district of Japan, near Tokyo.

Participants: A total of 1475 asymptomatic adults aged 30-79 years who underwent detailed medical check-ups and who regularly consumed small amounts of alcohol ( $<20 \mathrm{~g} /$ day $)$.

Outcome measures: Serum amylase, cardiometabolic risk factors, NAFLD determined by ultrasound, MetS determined by Adult Treatment Panel-III criteria and diabetes were assessed.

Results: The prevalence of NAFLD increased significantly from $22.5 \%$ to $42.4 \%$ (all grades) and from $9.2 \%$ to $24.0 \%$ (moderate or severe grade) from the highest to the lowest quartile of serum amylase. Multiple logistic regression analysis showed that, compared with the highest quartile of serum amylase, the lowest quartile of serum amylase was significantly associated with any-grade NAFLD and with moderateto-severe NAFLD, even after adjusting for MetS or diabetes. The association between LSA and any-grade NAFLD disappeared after further adjustment for body mass index or waist circumference, whereas the association between LSA and moderate or severe NAFLD remained statistically significant $(\mathrm{ORs}(95 \% \mathrm{Cl})$, 2.01 (1.07 to 3.78 ) and 2.06 (1.09 to 3.87 ), respectively, both $\mathrm{p}=0.01$ ).

Conclusions: Our results suggest that LSA may be associated with moderate or severe NAFLD in asymptomatic adults independent of MetS, diabetes and obesity. These results warrant confirmation in further studies.

\section{ARTICLE SUMMARY}

Article focus

- The clinical relevance of low serum amylase (LSA) is unclear, but it is putatively associated with obesity-related metabolic abnormalities.

- It is unknown as to whether LSA is associated with non-alcoholic fatty liver disease (NAFLD), a hepatic manifestation of cardiometabolic disease and insulin resistance.

Key messages

- Our results suggest that LSA is associated with NAFLD independent of metabolic syndrome, diabetes and obesity.

- LSA may be an independent marker for moderate or severe NAFLD.

Strengths and limitations of this study

- A possible association between LSA and NAFLD was evaluated after fully adjusting for relevant confounding factors.

- NAFLD was diagnosed with ultrasound in this study. Other methods, such as CT, MRI and fibroscan, may provide more precise assessment of NAFLD.

- This was an observational study and the causeeffect relationship is unknown.

\section{INTRODUCTION}

Abnormal serum amylase levels generally reflect the overall dysfunction of the pancreas or salivary glands. Although the clinical relevance of elevated serum amylase levels has been extensively studied in relation to various conditions, including acute pancreatitis, pancreatic cancer, ectopic amylaseproducing tumours, abdominal trauma and kidney dysfunction, ${ }^{1-5}$ the clinical relevance of low serum amylase (LSA) has not been examined. LSA is conventionally considered as a crude marker for diffuse pancreas 
destruction secondary to pancreatic diseases, such as advanced chronic pancreatitis and cystic fibrosis. ${ }^{6} 7$ LSA or low pancreatic amylase is also associated with insulin deficiency in patients with type 1 diabetes and in patients with longstanding type 2 diabetes. $^{8-11}$ In our recent community-based study, LSA, defined as a serum amylase concentration of $<60 \mathrm{IU} / 1$, was observed in $25 \%$ of asymptomatic individuals. ${ }^{12}$ LSA is also associated with metabolic syndrome (MetS), a cluster of cardiovascular risk factors, and diabetes. ${ }^{12} 13$ However, obesity is thought to be the strongest predictor of LSA in the asymptomatic general population. ${ }^{12}$

In the last two decades, there has been a marked increase in the prevalence of non-alcoholic fatty liver disease (NAFLD) worldwide, along with an obesity pandemic. $^{14} 15$ NAFLD often progresses to a more severe condition, non-alcoholic steatohepatitis (NASH) and increased hepatic fibrosis is a significant histological feature of advanced NASH. NAFLD is considered to be a hepatic manifestation of MetS and insulin resistance, ${ }^{14-18}$ which suggests that NAFLD consists of a wide spectrum of cardiometabolic diseases. It is also possible that NAFLD may reflect more pronounced insulin resistance compared with MetS in certain clinical settings, including in individuals without diabetes or obesity. ${ }^{17} 19{ }^{20}$ In this context, we hypothesised that LSA may be associated with NAFLD independently of MetS, type 2 diabetes, and even obesity. The aim of this study was to test this hypothesis and to investigate the clinical relevance of LSA, which is often observed in clinical practice. Therefore, we examined the associations among serum amylase, cardiometabolic risk factors, NAFLD, hepatic fibrosis, MetS and diabetes in a cross-sectional study of asymptomatic adults.

\section{METHODS}

\section{Protocol and subjects}

The present report represents a series of observational studies performed in collaboration with Josai University, Sakado, Japan and Social Insurance Omiya General Hospital that have been conducted to elucidate the relationships between lifestyle-related diseases and cardiometabolic risk factors. We recruited, at random, 2472 asymptomatic subjects aged 30-79 years who underwent thorough annual medical check-ups, in which the subjects underwent a more extensive array of clinical tests than would be performed in routine check-ups, at the Social Insurance Omiya General Hospital, Saitama, Japan, between April 2009 and March 2010. The protocol was approved by The Ethics Committee of Josai University and the Council of the Hospital, and informed consent was obtained from all participants.

The exclusion criteria and a flow chart summarising subject disposition are shown in online supplementary figure S1. To exclude subjects with latent conditions likely to adversely affect the results, we excluded those with $\mathrm{C}$ reactive protein $\geq 10.0 \mathrm{mg} / \mathrm{l}$, estimated glomerular filtration rate $(\mathrm{eGFR}) \leq 35 \mathrm{ml} / \mathrm{min} / 1.73 \mathrm{~m}^{2}$, serum amylase $\leq 30 \mathrm{IU} / 1$ (the lower fifth percentile in an earlier study $^{12}$ ) or $\geq 200 \mathrm{IU} / 1$ based on previous reports, ${ }^{21}{ }^{22}$ as well as subjects suspected of having cancer. In the current analysis, to investigate the potential relationship between serum amylase and cardiometabolic features, including NAFLD, we included subjects with a wider range of serum amylase levels than the current reference ranges.

Subjects completed a questionnaire recording lifestyle factors, including habitual alcohol consumption, which was classified in terms of the frequency (none, occasional and daily) and the amount of ethanol consumed per day $(<20,20-39,40-59$ or $\geq 60)$. Subjects who habitually consumed $\geq 20 \mathrm{~g}$ ethanol/day were excluded from the study. Subjects positive for hepatic B virus surface antigen were also excluded. Hepatic $\mathrm{C}$ virus infection was not measured in this study. We also excluded subjects with other active liver diseases not examined, by excluded those with elevated serum liver enzyme levels (approximately 3 times the upper limit of normal): alanine aminotransferase $($ ALT) $\geq 150 \mathrm{IU} / \mathrm{ml}$, aspartate aminotransferase (AST) $\geq 150 \mathrm{IU} / \mathrm{ml}$ or $\gamma$-glutamyltransferase (GGT) $\geq 150 \mathrm{IU} / \mathrm{ml}$. Consequently, a total of 1475 individuals were included in this study.

\section{Laboratory measurements}

Laboratory and anthropometric tests, and an abdominal ultrasound were performed in the early morning after an overnight fast. Serum parameters were measured using an AutoAnalyzer (Hitachi, Tokyo, Japan). The serum amylase level was measured using an enzymatic method (L-type Amylase; Wako, Tokyo, Japan) with a detection limit of $1.7 \mathrm{IU} / 1$, and a run-to-run coefficient of variation of $<5 \%$.

Abdominal ultrasound for the detection of fatty liver was carried out by registered medical sonographers who only work at Social Insurance Omiya General Hospital. The sonographers were blinded to the subjects' data. Fatty liver, which was determined by comparing liver echogenicity with that of the renal cortex, ${ }^{23}$ was defined as NAFLD. Additionally, the severity of NAFLD was graded into three categories: mild NAFLD, a slight increase in liver echogenicity with normal visualisation of the diaphragm and the portal veins; moderate NAFLD, a moderate increase in liver echogenicity with slightly impaired visualisation of the diaphragm and the portal veins; and severe NAFLD, a substantial increase in liver echogenicity with poor or no visualisation of the diaphragm and the portal veins. ${ }^{24} 25$ In a previous study, the ultrasonographic steatosis score determined using these grades was highly correlated with the histological grade of steatosis $(\mathrm{r}=0.80, \mathrm{p}<0.001)$, but not with inflammatory activity $(r=0.10)$ or fibrosis score $(r=0.19){ }^{25}$ Therefore, the grade of NAFLD in this study probably reflects overall hepatic fat accumulation, rather than the severity of fibrosis. Since it is possible that mild NAFLD could include a normal liver, we defined overt NAFLD as moderate or severe NAFLD. We also examined the findings of gallstones, cholecystectomy and splenomegaly, which was 
defined as a spleen index (calculated as the long dimension $\times$ short dimension on splenotomogram) $\geq 30 .{ }^{26} 27$

The liver fibrosis scores, the AST/ALT ratio ${ }^{28}$ and FIB-4 were calculated in all patients, using a previously published formula:

FIB-4=age (years) $\times$ AST $/$ platelet count $\left.\left(10^{9} / 1\right) \times \mathrm{ALT}^{1 / 2}\right){ }^{29}$

The diagnosis of MetS was based on the Adult Treatment Panel-III criteria ${ }^{30}$ with the following cut-off limits: (1) systolic blood pressure $\geq 130 \mathrm{~mm} \mathrm{Hg}$ or diastolic blood pressure $\geq 85 \mathrm{~mm} \mathrm{Hg}$; (2) triglyceride $\geq 150 \mathrm{mg} / \mathrm{dl}$; (3) high-density lipoprotein-cholesterol $<40 \mathrm{mg} / \mathrm{dl}$ for men and $<50 \mathrm{mg} / \mathrm{dl}$ for women; (4) fasting plasma glucose $\geq 100 \mathrm{mg} / \mathrm{dl}$ and (5) waist circumference (WC) $\geq 90 \mathrm{~cm}$ for men and $\geq 80 \mathrm{~cm}$ for women. Subjects meeting three or more of these criteria, including treatment for any of these disorders, were defined as having MetS. Diabetes was defined as fasting plasma glucose $\geq 126 \mathrm{mg} / \mathrm{dl}$ or glycosylated haemoglobin (HbA1c) $\geq 6.5 \%$ (by the National Glycoprotein Standardization Program (NGSP)) according to the American Diabetes Association criteria, ${ }^{31}$ or treatment with oral hypoglycaemic drugs or insulin. HbAlc (Japan Diabetes Society (JDS)) was converted to HbAlc (NGSP) units using the officially certified formula: HbAlc (NGSP) $(\%)=1.02 \times J \mathrm{JS}(\%)+0.25 \% .{ }^{32}$

Since serum amylase can be affected by kidney function because of its excretion by the kidney, ${ }^{4}$ eGFR was considered as a confounding factor and was calculated using the following equation for Japanese subjects: ${ }^{33}$ eGFR $\left(\mathrm{ml} / \mathrm{min} / 1.73 \mathrm{~m}^{2}\right)=194 \times$ serum $\mathrm{Cr}^{-1.094} \times$ age $^{-0.287}$ (if female) $\times 0.739$. Here, $\mathrm{Cr}=$ serum creatine concentration $(\mathrm{mg} / \mathrm{dl})$.

\section{Statistical analysis}

Data are expressed as the mean $\pm \mathrm{SD}$ or median (IR). Subjects were divided into quartiles according to serum amylase levels, where Q1 is the highest quartile and Q4 is the lowest quartile. $p$ Values for continuous variables were determined using analysis of variance or the Mann-Whitney U test, and for categorical variables using the $\chi^{2}$ test. Highly skewed values (ALT, AST, GGT and triglyceride) were log-transformed before analysis. Subjects were also stratified into four groups according to the grade of NAFLD (ie, normal, mild, moderate and severe NAFLD). Multivariate logistic regression models were used to examine the associations of quartiles (Q2-4) of serum amylase with any-grade NAFLD (NAFLD-AG), and with overt NAFLD relative to Q1, controlling for relevant confounding factors, including MetS, diabetes and body mass index (BMI) or WC. To elucidate any association between LSA and NAFLD, we examined the associations between LSA and NAFLD-AG, and between LSA and overt NAFLD. This analysis yielded OR and 95\% CI. Tests for linear trends ( $p$ for trend) were calculated by treating the quartiles as a continuous variable (ie, 1-4), and the same model analysis was conducted. Statistical analyses were performed using SPSS software V.18.0 (SPSS-IBM, Chicago, Illinois, USA) and Statview
V.5.0 (SAS Institute, Cary, North Carolina, USA). Values of $\mathrm{p}<0.05$ were considered to be statistically significant.

\section{RESULTS}

The clinical characteristics of the subjects according to the quartile of serum amylase are shown in table 1 . The mean and median values of most clinical parameters, including platelet count and eGFR, increased significantly as serum amylase decreased, although there was a significant trend for subjects with lower amylase levels to be younger. No significant trend in HbAlc was observed against quartiles of serum amylase. The prevalence of NAFLD-AG and overt NAFLD increased significantly, from $22.5 \%$ to $42.4 \%$ and from $9.2 \%$ to $24 \%$, respectively, from the highest $(\mathrm{Q} 1)$ to the lowest serum amylase quartile $(\mathrm{Q} 4)$. There were no significant differences in the prevalence rates of gallstones, cholecystectomy or splenomegaly between quartiles, possibly because of the small numbers of subjects with these features, although the rates of cholecystectomy and splenomegaly were higher in Q4 compared with the other quartiles. Log-transformed serum ALT and GGT increased significantly, whereas the AST/ALT ratio and FIB-4 decreased significantly with decreasing serum amylase quartile.

When subjects were stratified according to NAFLD grade, serum amylase decreased significantly with advancing grade of NAFLD (figure 1). When subjects were further stratified according to sex, there was no significant difference between men and women. However, when the subjects were stratified according to high or low BMI (figure 2), serum amylase levels were significantly lower in overweight/obese subjects (BMI $\geq 25 \mathrm{~kg} / \mathrm{m}^{2}$, mean \pm SD BMI, $\left.27.3 \pm 2.3 \mathrm{~kg} / \mathrm{m}^{2}, \mathrm{n}=1121\right)$ than in lean subjects $\left(\mathrm{BMI}<25 \mathrm{~kg} / \mathrm{m}^{2}\right.$, mean \pm SD BMI, $21.6 \pm 2 \mathrm{~kg} / \mathrm{m}^{2}$, $\mathrm{n}=354$ ). Serum amylase levels in lean subjects decreased significantly with advancing grade of NAFLD, whereas those in obese subjects did not. Significant differences between lean and obese subjects were only observed in subjects with a normal liver or mild NAFLD. We did not conduct an analysis by further stratification according to MetS or diabetes because of the small proportion of subjects (both, $<10 \%$ ).

Multiple logistic regression analysis showed that, compared with the highest quartile of serum amylase ( $\geq 90 \mathrm{IU} / \mathrm{l}, \mathrm{Q} 1)$, the lowest quartile $(<60 \mathrm{IU} / 1, \mathrm{Q} 4)$ was significantly associated with NAFLD-AG even after adjusting for confounders plus MetS or diabetes (table 2, Models 4 and 5). In these conditions, MetS (OR 3.66, 95\% CI 2.37 to 5.65) and diabetes (OR 1.96, 95\% CI 1.25 to3.05) were significantly associated with Q4 of serum amylase (data not shown). However, these associations were markedly attenuated and were no longer statistically significant after adjusting for clinical confounders plus BMI or WC (Models 6 and 7), although there was a trend towards an association among the quartiles of serum amylase.

When we excluded subjects with mild NAFLD $(n=242)$ and repeated the analysis (table 3 ), we detected stronger 
Table 1 Characteristics of subjects stratified according to quartiles of serum amylase

\begin{tabular}{|c|c|c|c|c|c|c|}
\hline & Total & Q1 (highest) & Q2 & Q3 & Q4 (lowest) & p Value \\
\hline$n$ & 1475 & 369 & 371 & 381 & 354 & \\
\hline Age (years) & $55.1 \pm 12.2$ & $58.4 \pm 11.8$ & $56.4 \pm 12.2$ & $52.9 \pm 11.7$ & $52.7 \pm 12.0$ & $<0.0001$ \\
\hline Men, n (\%) & $747(50.6)$ & $178(48.2)$ & $176(47.4)$ & $205(53.8)$ & $188(53.1)$ & 0.19 \\
\hline $\mathrm{BMI}\left(\mathrm{kg} / \mathrm{m}^{2}\right)$ & $23.0 \pm 3.2$ & $21.9 \pm 2.7$ & $22.6 \pm 3.2$ & $23.3 \pm 3.1$ & $24.1 \pm 3.4$ & $<0.0001$ \\
\hline WC $(\mathrm{cm})$ & $80.8 \pm 8.9$ & $78.2 \pm 8.3$ & $79.9 \pm 8.8$ & $81.8 \pm 8.8$ & $83.2 \pm 9.1$ & $<0.0001$ \\
\hline Systolic blood pressure $(\mathrm{mm} \mathrm{Hg})$ & $120 \pm 18.9$ & $119 \pm 18.5$ & $119 \pm 18.7$ & $120 \pm 18.9$ & $123 \pm 19.1$ & 0.007 \\
\hline Diastolic blood pressure (mm Hg) & $74.1 \pm 12.6$ & $72.6 \pm 12.2$ & $73.1 \pm 11.9$ & $74.5 \pm 13.4$ & $76.1 \pm 12.4$ & 0.0009 \\
\hline Platelet count $\left(10^{9} / \mathrm{l}\right)$ & $235 \pm 53.4$ & $228 \pm 53.8$ & $235 \pm 53.1$ & $237 \pm 51.1$ & $241 \pm 55.2$ & 0.01 \\
\hline ALT (IU/I) & $18(14-25)$ & $18(14-23)$ & $17(14-23)$ & $18(14-25)$ & 19 (14-29) & $<0.0001$ \\
\hline AST (IU/I) & $20(17-24)$ & $21(19-25)$ & $20(17-24)$ & $19(17-23)$ & $20(17-24)$ & 0.04 \\
\hline AST/ALT & $1.15 \pm 0.36$ & $1.24 \pm 0.35$ & $1.20 \pm 0.37$ & $1.12 \pm 0.33$ & $1.04 \pm 0.35$ & $<0.0001$ \\
\hline FIB-4 & $1.13(0.82-1.52)$ & $1.33(0.98-1.76)$ & $1.20(0.89-1.59)$ & $1.05(0.76-1.38)$ & $0.98(0.70-1.36)$ & $<0.0001$ \\
\hline GGT (IU/I) & $22(16-33)$ & $20(15-31)$ & $20(16-30)$ & $23(16-34)$ & $24(17-38)$ & 0.0009 \\
\hline $\mathrm{C}$ reactive protein & $0.4(0.3-0.8)$ & $0.3(0.3-0.63)$ & $0.4(0.3-0.8)$ & $0.4(0.3-0.8)$ & $0.5(0.3-1.1)$ & $<0.0001$ \\
\hline Amylase (IU/l) (range) & $77.3 \pm 24.9(30-200)$ & $110 \pm 22.0(90-200)$ & $81.3 \pm 4.7(74-89)$ & $66.6 \pm 4.1(60-73)$ & $50.3 \pm 7.0(30-59)$ & - \\
\hline Triglyceride (mg/dl) & $88(64-128)$ & $83(63-121)$ & $84(62-123)$ & $88(65-128)$ & $100(65-143)$ & 0.001 \\
\hline HDL cholesterol (mg/dl) & $61.2 \pm 15.0$ & $64.2 \pm 15.4$ & $62.6 \pm 14.8$ & $59.8 \pm 14.6$ & $58.3 \pm 14.5$ & $<0.0001$ \\
\hline Fasting plasma glucose (mg/dl) & $99.6 \pm 16.7$ & $97.5 \pm 13.4$ & $97.4 \pm 11.4$ & $101 \pm 19.4$ & $103 \pm 20.4$ & $<0.0001$ \\
\hline HbA1c (NGSP) (\%) & $5.7 \pm 0.6$ & $5.7 \pm 0.5$ & $5.7 \pm 0.4$ & $5.7 \pm 0.6$ & $5.8 \pm 0.7$ & 0.14 \\
\hline eGFR $\left(\mathrm{ml} / \mathrm{min} / 1.73 \mathrm{~m}^{2}\right)$ & $75.0 \pm 13.9$ & $71.0 \pm 14.3$ & $74.3 \pm 12.9$ & $76.0 \pm 14.2$ & $78.7 \pm 13.1$ & $<0.0001$ \\
\hline ATP-III-metabolic syndrome, n (\%) & $139(9.4)$ & $16(4.3)$ & $23(6.2)$ & $47(12.3)$ & $53(15.0)$ & $<0.0001$ \\
\hline Diabetes, n (\%) & $114(7.7)$ & $19(5.1)$ & $17(4.6)$ & $34(8.9)$ & $44(12.4)$ & 0.0002 \\
\hline NAFLD of all grades, $n(\%)$ & $463(31.4)$ & $83(22.5)$ & $97(26.1)$ & $133(34.9)$ & $150(42.4)$ & $<0.0001$ \\
\hline Moderate-to-severe NAFLD, n (\%) & $221(15.0)$ & $34(9.2)$ & $42(11.3)$ & $60(15.7)$ & $85(24.0)$ & $<0.0001$ \\
\hline Gallstones, $\mathrm{n}(\%)$ & $81(5.5)$ & $21(5.7)$ & $20(5.4)$ & $24(6.3)$ & $16(5.2)$ & 0.76 \\
\hline Cholecystectomy, n (\%) & $23(1.6)$ & $5(1.4)$ & $3(0.8)$ & $5(1.3)$ & $10(2.8)$ & 0.15 \\
\hline Splenomegaly, $\mathrm{n}(\%)$ & $9(0.6)$ & $1(0.3)$ & $1(0.3)$ & 0 & $7(2.0)$ & $-^{*}$ \\
\hline \multicolumn{7}{|l|}{ Medical history of } \\
\hline Cardiovascular diseases, n (\%) & $55(3.7)$ & $11(3.0)$ & $20(5.4)$ & $11(2.9)$ & $13(3.7)$ & 0.24 \\
\hline Stroke, $n(\%)$ & $26(1.8)$ & $12(3.3)$ & $6(1.6)$ & $4(1.1)$ & $4(1.1)$ & 0.08 \\
\hline \multicolumn{7}{|l|}{ Medications for } \\
\hline Hypertension, n (\%) & $237(16.1)$ & $64(17.3)$ & $47(12.7)$ & $67(17.6)$ & $59(16.7)$ & 0.22 \\
\hline Hypercholesterolaemia, n (\%) & $177(12.0)$ & $56(15.2)$ & $43(11.6)$ & $34(8.9)$ & $44(12.4)$ & 0.07 \\
\hline Diabetes, n (\%) & $56(3.8)$ & $12(3.3)$ & $12(3.2)$ & $15(3.9)$ & $17(4.8)$ & 0.65 \\
\hline Current smoker, n (\%) & $273(18.5)$ & $40(10.8)$ & $50(13.5)$ & $86(22.6)$ & $97(27.4)$ & $<0.0001$ \\
\hline Everyday alcohol consumers, n (\%) & $229(15.5)$ & $51(13.8)$ & 59 (15.9) & $66(17.3)$ & $53(15.0)$ & 0.61 \\
\hline Regular exercise, n (\%) & $507(34.4)$ & $144(39.0)$ & $135(36.4)$ & $125(32.9)$ & $103(29.1)$ & 0.03 \\
\hline
\end{tabular}

Data are means \pm SD and medians (IR). $p$ Values for continuous variables and categorical variables were determined by analysis of variance and the $\chi^{2}$ test, respectively. Regular exercise: $\geq 30$ min exercise per session at least twice a week.

* Statistical analysis was not performed because of the small number of subjects with splenomegaly.

ALT, alanine aminotransferase; AST, aspartate aminotransferase; BMI, body mass index; GGT, $\gamma$-glutamyltransferase; HDL, high-density lipoprotein; NAFLD, non-alcoholic fatty liver disease; NGSP, National Glycoprotein Standardization Program; WC, waist circumference. 


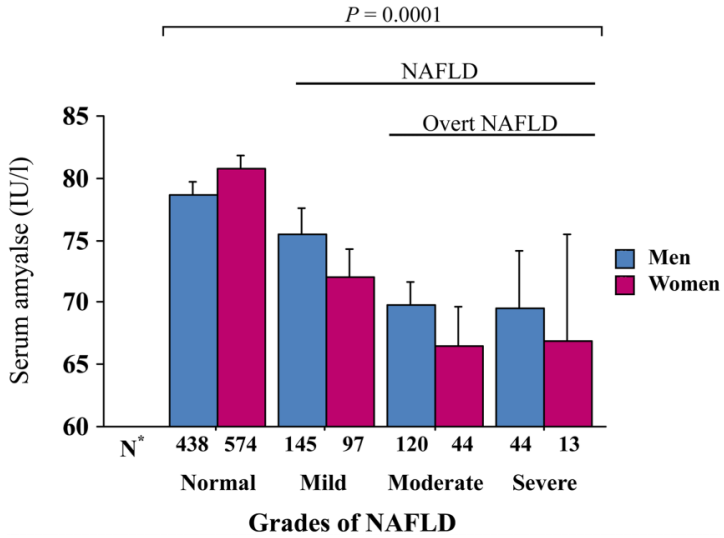

Figure 1 Serum amylase levels according to the grade of non-alcoholic fatty liver disease (NAFLD) and sex. Serum amylase levels decreased significantly with advancing grade of NAFLD in both sexes (analysis of variance (ANOVA), both $p=0.0001$ ). There was no significant difference between men and women (two-way ANOVA, $p=0.45$ ). *Number of subjects in each group. Bars represent SEs.

significant associations between Q4 and overt NAFLD. These associations were also moderately attenuated after additional adjustment for BMI or WC, but they remained statistically significant (Models 6 and 7). In this condition, BMI (OR 1.65, 95\% CI 1.51 to 1.80 ) and WC (OR 1.21, 95\% CI 1.17 to 1.25 ) were significantly associated with overt NAFLD (data not shown).

\section{DISCUSSION}

For the last few decades, NAFLD has been considered as a manifestation of MetS and insulin resistance. ${ }^{14-18}$ In

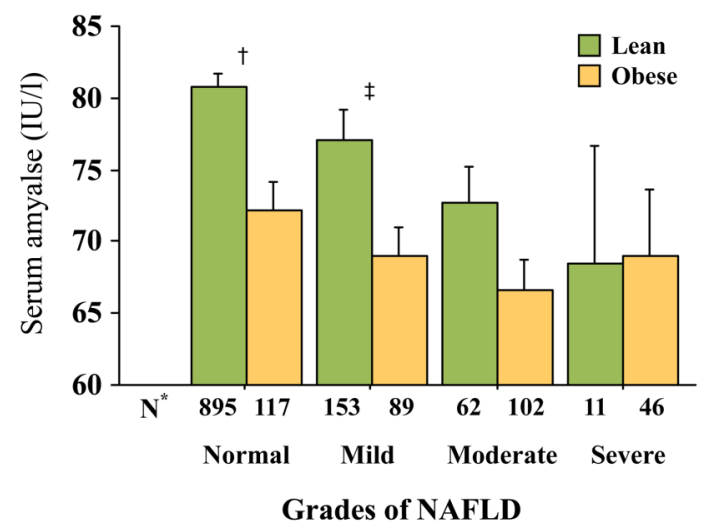

Figure 2 Serum amylase levels according to the grade of non-alcoholic fatty liver disease (NAFLD) and obesity. Obesity was defined as body mass index $\geq 25 \mathrm{~kg} / \mathrm{m}^{2}$. Serum amylase levels decreased significantly with advancing grade of NAFLD in lean subjects (analysis of variance (ANOVA), $p=0.01$ ), but not in obese subjects $(p=0.33)$. An overall significant difference was observed between lean and obese subjects (two-way ANOVA, $\mathrm{p}=0.03$ ). *Number of subjects in each group; ${ }^{\dagger} p=0.0001$ and ${ }^{\ddagger} p=0.02$ for lean versus obese subjects (Mann-Whitney U test). Bars represent SEs. this context, the present study showed that LSA was significantly associated with NAFLD-AG in asymptomatic adults, independent of MetS, diabetes and sex. Furthermore, LSA may be indicative of overt NAFLD, that is, moderate or severe NAFLD, independent of obesity. These findings are not inconsistent because NAFLD may reflect insulin resistance, more so than MetS, in individuals without diabetes or obesity. ${ }^{17} 1920$

This study provided evidence that NAFLD can occur in lean individuals, who accounted for $37.8 \%(62 / 104)$ of subjects with moderate NAFLD and $19.3 \%(11 / 57)$ of subjects with severe NAFLD (figure 2). This is consistent with the results of multivariate logistic analysis, where there was a significant trend for NAFLD against quartiles of serum amylase, and the significant association between the lowest quartile of serum amylase and NAFLD remained after adjusting for BMI and WC (tables 2 and 3).

\section{Potential mechanisms of the inverse relationship between} serum amylase and NAFLD

We found no significant difference in serum amylase levels between lean and obese subjects with overt NAFLD, suggesting that the effects of obesity on serum amylase are apparent in subjects with a normal liver or with mild NAFLD, but not in subjects with overt NAFLD. Several studies have shown that NAFLD occurs in nonobese people, although the prevalence of NAFLD in non-obese subjects is lower than that in obese subjects. ${ }^{19} 2034$ Therefore, other than obesity, factors not examined in this study might also explain the association between LSA and NAFLD. Insulin resistance and obesity-related hormones, such as leptin, may be potential factors, because insulin resistance and energy imbalance are common pathophysiological findings in obese individuals. ${ }^{17} 35$ Indeed, obesity plus insulin resistance is more closely associated with metabolic abnormalities and NAFLD than is obesity without insulin resistance. ${ }^{17}$ Hyperinsulinaemia caused by insulin resistance stimulates de novo lipogenesis in the liver through sterol regulatory element binding protein-1c (SREBP-1c), ${ }^{36}$ irrespective of the influx of fatty acid from a meal and lipolysis in adipose tissues. Additionally, we recently reported a latent association between LSA and insulin resistance in a small study of asymptomatic middle-aged adults, ${ }^{37}$ supporting the hypothesis that LSA may be associated with NAFLD, at least in part through insulin resistance. However, because insulin resistance, as determined by the homeostasis model assessment of insulin resistance, for example, was not examined in this study, further large epidemiological studies evaluating insulin resistance are needed to confirm the current hypothesis.

As another explanation for the inverse relationship between serum amylase and NAFLD, systemic ectopic fat deposition in organs including the pancreas (ie, fatty pancreas) might contribute to the observed associations. This is because intrapancreatic fat, particularly intralobular pancreatic fat, was reported to be associated with 
Table 2 ORs for NAFLD-AG according to serum amylase quartiles

\begin{tabular}{|c|c|c|c|c|c|}
\hline Serum amylase quartiles & Q1 (highest) & Q2 & Q3 & Q4 (lowest) & $\bar{p}$ for trend \\
\hline$N$ & 369 & 371 & 381 & 354 & \\
\hline Model 1 & 1 & $1.22(0.87$ to 1.71$)$ & 1.85 (1.34 to 2.55$)$ & 2.53 (1.84 to 3.50$)$ & $<0.0001$ \\
\hline Model 2 & 1 & $1.28(0.91$ to 1.81$)$ & 1.96 (1.39 to 2.74$)$ & 2.74 (1.95 to 3.85$)$ & $<0.0001$ \\
\hline Model 3 & 1 & $1.35(0.93$ to 1.97$)$ & 2.03 (1.41 to 2.94$)$ & 2.41 (1.66 to 3.51$)$ & $<0.0001$ \\
\hline Model 4 & 1 & $1.25(0.86$ to 1.82$)$ & $1.83(1.26$ to 2.65$)$ & $2.18(1.49$ to 3.18$)$ & $<0.0001$ \\
\hline Model 5 & 1 & $1.31(0.90$ to 1.90$)$ & $2.00(1.39$ to 2.89$)$ & $2.36(1.62$ to 3.44$)$ & $<0.0001$ \\
\hline Model 6 & 1 & 0.99 (0.66 to 1.49$)$ & $1.34(0.89$ to 2.01$)$ & $1.42(0.94$ to 2.15$)$ & 0.04 \\
\hline Model 7 & 1 & $1.02(0.68$ to 1.53$)$ & $1.30(0.87$ to 1.94$)$ & $1.49(0.99$ to 2.24$)$ & 0.03 \\
\hline
\end{tabular}

Model 1: unadjusted.

Model 2: adjusted for age, sex and current smoking (vs non-smokers).

Model 3: Model 2 plus adjustment for log-transformed ALT, regular exercise (vs infrequent exercise), daily alcohol consumption (vs infrequent/ no alcohol consumption), eGFR, history of heart diseases and stroke and medications for hypertension, diabetes and dyslipidaemia.

Model 4: Model 3 plus adjustment for diabetes, but excluding medications for hypertension, diabetes and dyslipidaemia.

Model 5: Model 3 plus adjustment for metabolic syndrome, but excluding medications for hypertension, diabetes and dyslipidaemia.

Model 6: Model 3 plus adjustment for BMI.

Model 7: Model 3 plus adjustment for WC.

ALT, alanine aminotransferase; BMI, body mass index; eGFR, estimated glomerular filtration rate; NAFLD, non-alcoholic fatty liver disease;

WC, waist circumference.

NAFLD and MetS. ${ }^{38} 39$ An animal study showed that obese mice had a heavier pancreas and more intrapancreatic fat. ${ }^{40}$ Therefore, systemic fat deposits in multiple organs may be a common cause underlying the association between NAFLD and overall pancreatic dysfunction, which may result in impaired exocrine function, characterised as LSA.

Gene polymorphisms of patatin-like phospholipase domain containing 3 (PNPLA3) may also contribute to the strength of these associations. For example, a common variant of the PNPLA3 gene (rs738409) was reported to be associated with increased hepatic fat content (ie, NAFLD). ${ }^{41}$ In the mouse liver, adiponutrin/ PNPLA3 gene expression is under the direct transcriptional control of SREBP-1c, in response to insulin. ${ }^{42}$

\section{Serum amyalse and glucose metabolism}

The prevalence of diabetes increased with decreasing serum amylase quartile in this study. However, similar to an earlier study, ${ }^{12} \mathrm{HbA1c}$ was not associated with LSA, probably because most subjects in the lowest quartile of serum amylase in this study had no or only mild insulin resistance or mild hyperinsulinaemia, which is likely compensated for and results in euglycaemia or mild hyperglycaemia. In such conditions, fasting plasma glucose would increase linearly, as observed in table 1, in response to insulin resistance, particularly in the early stage of diabetes. ${ }^{43} 44$

\section{Serum amyalse and hepatic fibrosis}

To date, some markers have been considered for screening for NAFLD and mild hepatic fibrosis. ${ }^{45-47}$ In this study, while the AST/ALT ratio and Fib-4 decreased significantly with the decreasing quartile of serum amylase, the platelet count increased (table 1). Consequently, hepatic fibrosis is unlikely to be associated with NAFLD in this study. A plausible explanation is that this study mostly consisted of non-obese individuals, with a small proportion having MetS and diabetes, resulting in a lower likelihood of advanced hepatic fibrosis and NASH. In fact, the estimated prevalence of NASH in this study is quite low when we compare it with the prevalence of NASH in a nationwide study in Japan. It was reported that approximately $20-25 \%$ of patients with diabetes had NAFLD, a population in which the prevalence of NASH might be $30-40 \% .{ }^{48}$ Therefore, the estimated prevalence

Table 3 ORs of each serum amylase for NAFLD of moderate or severe grade

\begin{tabular}{|c|c|c|c|c|c|}
\hline Serum amylase quartiles & Q1 (highest) & Q2 & Q3 & Q4 (lowest) & $p$ for trend \\
\hline $\mathrm{N}^{*}$ & 320 & 316 & 308 & 289 & \\
\hline Model 1 & 1 & 1.29 (0.80 to 2.09$)$ & 2.04 (1.29 to 3.20$)$ & 3.51 (2.27 to 5.42$)$ & $<0.0001$ \\
\hline Model 2 & 1 & $1.36(0.83$ to 2.22$)$ & $2.14(1.33$ to 3.44$)$ & $3.81(2.40$ to 6.05$)$ & $<0.0001$ \\
\hline Model 3 & 1 & $1.61(0.91$ to 2.86$)$ & 2.32 (1.32 to 4.07$)$ & 3.45 (1.99 to 6.00$)$ & $<0.0001$ \\
\hline Model 4 & 1 & $1.48(0.84$ to 2.64$)$ & 2.11 (1.19 to 3.73$)$ & $2.97(1.70$ to 5.20$)$ & $<0.0001$ \\
\hline Model 5 & 1 & 1.56 (0.89 to 2.75$)$ & 2.32 (1.33 to 4.04$)$ & 3.26 (1.88 to 5.66$)$ & $<0.0001$ \\
\hline Model 6 & 1 & 1.05 (0.54 to 2.03$)$ & 1.44 (0.76 to 2.72$)$ & 2.01 (1.07 to 3.78$)$ & 0.01 \\
\hline Model 7 & 1 & $1.06(0.55$ to 2.05$)$ & $1.37(0.73$ to 2.59$)$ & 2.06 (1.09 to 3.87$)$ & 0.01 \\
\hline
\end{tabular}

*Subjects with mild NAFLD $(n=242)$ were excluded from this analysis. Models 1-7 are the same as those in table 2.

NAFLD, non-alcoholic fatty liver disease. 
of NASH might be less than $1 \%$ of all of the subjects in this study. In addition, the fact that more than half of the subjects in this study had repeatedly undergone detailed medical check-ups may also contribute to the lower prevalence of NASH, because repeated check-ups may promote consciousness of healthcare and favourable lifestyles.

\section{LIMITATIONS}

Several limitations should be mentioned. First, NAFLD was diagnosed by ultrasound rather than by histological examination, the gold standard technique for the diagnosis of NAFLD. Thus, NAFLD and the degree of hepatic fibrosis could be inaccurately evaluated, particularly in the early stages. In earlier studies, ultrasonography had a sensitivity of $60-94 \%$ and a specificity of $66-95 \%$ for detecting fatty liver. ${ }^{24}{ }^{49}$ However, its sensitivity is reduced in subjects with a small amount of fat $(<30 \%)$, such as those with mild NAFLD or advanced fibrosis. ${ }^{14}$ To improve the accuracy of detecting and grading of NAFLD, the use of other imaging modalities might be needed, such as MRI and MR spectroscopy, which were reported to provide useful quantitative data in earlier studies. $^{24} 50$

Second, hepatitis $\mathrm{C}$ virus infection was not measured in this study. However, the prevalence of hepatitis C infection was reported to be $1.5-2.3 \%$ in Japan. ${ }^{51}$ Low prevalence rates of hepatitis $\mathrm{C}(<1.5 \%)$ were also recently reported in Asian-Pacific, tropical Latin American and North American countries. ${ }^{52}$ Therefore, hepatitis C infection is unlikely to profoundly modify the association between LSA and NAFLD in this study, although hepatitis C infection can contribute to the development of NAFLD. ${ }^{53}$ It is also possible that individuals with primary biliary cirrhosis, autoimmune hepatitis or other forms of liver dysfunction (eg, haemochromatosis and Wilson disease) were included in the present study, even though we excluded subjects with elevated hepatic enzymes $(\geq 150 \mathrm{IU} / \mathrm{ml})$. However, the prevalence of these diseases is quite low in the general population ${ }^{54-56}$

Third, because of the cross-sectional nature of the study, we could not determine the cause-effect relationship between LSA and NAFLD. However, in a previous retrospective study, ${ }^{12}$ subjects with LSA at baseline were more likely to develop MetS-related metabolic abnormalities 5 years later. Longitudinal prospective studies or clinical intervention trials are needed to elucidate the causality of the associations reported here.

Finally, the clinical relevance of measuring serum amylase remains unclear. The current results suggest that LSA is likely to detect overt NAFLD, but not NASH, which is the most important hepatic disorder. However, from the cardiometabolic perspective, even simple steatosis may be important, particularly in non-obese people, because steatosis may be more strongly associated with insulin resistance than is obesity. ${ }^{17} 1920$ Currently, serum amylase is rarely considered in clinical practice, except in certain situations, such as suspected pancreatitis. Therefore, numerous clinical and animal studies are needed to validate and confirm the clinical relevance of measuring serum amylase before it can be introduced into primary care for the detection of cardiometabolic diseases and NAFLD.

\section{CONCLUSION}

Our results suggest that LSA may be associated with NAFLD, particularly moderate or severe NAFLD, in asymptomatic adults. This association was independent of MetS, diabetes and obesity. Further studies are needed to confirm the observed associations, and to explore the clinical relevance of LSA.

\section{Author affiliations}

${ }^{1}$ Division of Clinical Nutrition, Department of Medical Dietetics, Josai University, Sakado, Saitama, Japan

${ }^{2}$ First Department of Comprehensive Medicine, Saitama Medical Center, Jichi Medical University School of Medicine, Omiya, Saitama, Japan

${ }^{3}$ Department of Health Care Center, Social Insurance Omiya General Hospital, Kita, Saitama, Japan

${ }^{4}$ Department of Internal Medicine, Social Insurance Omiya General Hospital, Kita, Saitama, Japan

Contributors KN, HM and MK designed the collaborative project; KN, HO, TM and $\mathrm{HF}$ collected and analysed the data; $\mathrm{KN}, \mathrm{HO}, \mathrm{MS}$ and $\mathrm{YH}$ researched and evaluated the literature and KN wrote the first draft of the manuscript. All authors reviewed and edited the manuscript, and approved the final version of the manuscript.

Funding This research received no specific grant from any funding agency in the public, commercial or not-for-profit sectors.

\section{Competing interests None.}

Patient consent Obtained.

Ethics approval The Ethics Committee of Josai University.

Provenance and peer review Not commissioned; externally peer reviewed.

Data sharing statement There are no additional data available.

\section{REFERENCES}

1. Yegneswaran B, Pitchumoni CS,, Yegneswaran B, et al. When should serum amylase and lipase levels be repeated in a patient with acute pancreatitis? Cleve Clin J Med 2010;77:230-1.

2. Skrha J, Stěpán J. Clinical significance of amylase isoenzyme determination. Acta Univ Carol Med Monogr 1987;120:1-81.

3. Pieper-Bigelow C, Strocchi A, Levitt MD. Where does serum amylase come from and where does it go? Gastroenterol Clin North Am 1990;19:793-810.

4. Junge W, Mályusz M, Ehrens HJ. The role of the kidney in the elimination of pancreatic lipase and amylase from blood. J Clin Chem Clin Biochem 1985;23:387-92.

5. Collen MJ, Ansher AF, Chapman AB, et al. Serum amylase in patients with renal insufficiency and renal failure. Am J Gastroenterol 1990;85:1377-80.

6. Domínguez-Muñoz JE, Pieramico O, Büchler M, et al. Ratios of different serum pancreatic enzymes in the diagnosis and staging of chronic pancreatitis. Digestion 1993;54:231-6.

7. Wolf RO, Hubbard VS, Gillard BK, et al. Three methods compared for determination of pancreatic and salivary amylase activity in serum of cystic fibrosis patients. Clin Chem 1986;32:296-300.

8. Frier BM, Faber OK, Binder C, et al. The effect of residual insulin secretion on exocrine pancreatic function in juvenile-onset diabetes mellitus. Diabetologia 1978;14:301-4.

9. Dandona P, Freedman DB, Foo Y, et al. Exocrine pancreatic function in diabetes mellitus. J Clin Pathol 1984;37:302-6. 
10. Swislocki A, Noth R, Hallstone A, et al. Secretin-stimulated amylase release into blood is impaired in type 1 diabetes mellitus. Horm Metab Res 2005;37:326-30.

11. Aughsteen AA, Abu-Umair MS, Mahmoud SA. Biochemical analysis of serum pancreatic amylase and lipase enzymes in patients with type 1 and type 2 diabetes mellitus. Saudi Med J 2005;26:73-7.

12. Nakajima K, Nemoto T, Muneyuki T, et al. Low serum amylase in association with metabolic syndrome and diabetes: a community-based study. Cardiovasc Diabetol 2011;10:34.

13. Lee JG, Park SW, Cho BM, et al. Serum amylase and risk of the metabolic syndrome in Korean adults. Clin Chim Acta 2011;412:1848-53.

14. Neuschwander-Tetri BA, Caldwell SH. Nonalcoholic steatohepatitis: summary of an AASLD Single Topic Conference. Hepatology 2003;37:1202-19.

15. Smith BW, Adams LA. Non-alcoholic fatty liver disease. Crit Rev Clin Lab Sci 2011;48:97-113.

16. Stefan $\mathrm{N}$, Häring $\mathrm{HU}$. The metabolically benign and malignant fatty liver. Diabetes 2011;60:2011-17.

17. Stefan N, Kantartzis K, Machann J, et al. Identification and characterization of metabolically benign obesity in humans. Arch Intern Med 2008;168:1609-16.

18. Angelico $\mathrm{F}$, Del Ben M, Conti $\mathrm{R}$, et al. Insulin resistance, the metabolic syndrome, and nonalcoholic fatty liver disease. J Clin Endocrinol Metab 2005;90:1578-82.

19. Kim HJ, Kim HJ, Lee KE, et al. Metabolic significance of nonalcoholic fatty liver disease in nonobese, nondiabetic adults. Arch Intern Med 2004;164:2169-75.

20. Musso G, Gambino R, Bo S, et al. Should nonalcoholic fatty liver disease be included in the definition of metabolic syndrome? A cross-sectional comparison with Adult Treatment Panel III criteria in nonobese nondiabetic subjects. Diabetes Care 2008;31:562-8.

21. Keogh JB, McGeeney KF, Drury MI, et al. Renal clearance of pancreatic and salivary amylase relative to creatinine in patients with chronic renal insufficiency. Gut 1978;19:1125-30.

22. Okura $\mathrm{Y}$, Hayashi K, Shingu $\mathrm{T}$, et al. Diagnostic evaluation of acute pancreatitis in two patients with hypertriglyceridemia. World $J$ Gastroenterol 2004;10:3691-5.

23. Tchelepi H, Ralls PW, Radin R, et al. Sonography of diffuse liver disease. J Ultrasound Med 2002;21:1023-32.

24. Schwenzer NF, Springer F, Schraml C, et al. Non-invasive assessment and quantification of liver steatosis by ultrasound, computed tomography and magnetic resonance. J Hepatol 2009:51:433-45.

25. Shannon A, Alkhouri N, Carter-Kent C, et al. Ultrasonographic quantitative estimation of hepatic steatosis in children with NAFLD. $J$ Pediatr Gastroenterol Nutr 2011;53:190-5.

26. Ishibashi $\mathrm{H}$, Higuchi N, Shimamura R, et al. Sonographic assessment and grading of spleen size. J Clin Ultrasound 1991;19:21-5.

27. Kataoka M, Nakata $Y$, Maeda $T$, et al. Ultrasonographic analysis of splenomegaly in patients with sarcoidosis. Nihon Kyobu Shikkan Gakkai Zasshi 1990;28:750-5. (In Japanese)

28. lacobellis A, Marcellini M, Andriulli A, et al. Non invasive evaluation of liver fibrosis in paediatric patients with nonalcoholic steatohepatitis. World J Gastroenterol 2006;12:7821-5.

29. Vallet-Pichard A, Mallet V, Nalpas B, et al. FIB-4: an inexpensive and accurate marker of fibrosis in HCV infection. Hepatology 2007;46:32-6.

30. Grundy SM, Cleeman JI, Daniels SR, et al. Diagnosis and management of the metabolic syndrome: an American Heart Association/National Heart, Lung, and Blood Institute Scientific Statement. Circulation 2005:112:2735-52.

31. American Diabetes Association. Diagnosis and classification of diabetes mellitus. Diabetes Care 2010;33(Suppl 1):S62-9.

32. Kashiwagi A, Kasuga M, Araki E, et al. International clinical harmonization of glycated hemoglobin in Japan: from Japan Diabetes Society to National Glycohemoglobin Standardization Program values. J Diabetes Investig 2012:3:39-40.
33. Matsuo S, Imai E, Horio M, et al. Revised equations for estimated GFR from serum creatinine in Japan. Am J Kidney Dis 2009;53:982-92.

34. Das K, Das K, Mukherjee PS, et al. Nonobese population in a developing country has a high prevalence of nonalcoholic fatty liver and significant liver disease. Hepatology 2010;51:1593-602.

35. Gautron L, Elmquist JK. Sixteen years and counting: an update on leptin in energy balance. J Clin Invest 2011;121:2087-93.

36. Dentin R, Pégorier JP, Benhamed F, et al. Hepatic glucokinase is required for the synergistic action of ChREBP and SREBP-1c on glycolytic and lipogenic gene expression. J Biol Chem 2004;279:20314-26.

37. Muneyuki T, Nakajima K, Aoki A, et al. Latent associations of low serum amylase with decreased plasma insulin levels and insulin resistance in asymptomatic middle-aged adults. Cardiovasc Diabeto 2012;11:80.

38. van Geenen EJ, Smits MM, Schreuder TC, et al. Nonalcoholic fatty liver disease is related to nonalcoholic fatty pancreas disease. Pancreas 2010;39:1185-90.

39. Sepe PS, Ohri A, Sanaka S, et al. A prospective evaluation of fatty pancreas by using EUS. Gastrointest Endosc 2011;73:987-93.

40. Mathur A, Marine M, Lu D, et al. Nonalcoholic fatty pancreas disease. HPB (Oxford) 2007;9:312-18.

41. Sookoian S, Pirola CJ. Meta-analysis of the influence of I148M variant of patatin-like phospholipase domain containing 3 gene (PNPLA3) on the susceptibility and histological severity of nonalcoholic fatty liver disease. Hepatology 2011;53:1883-94.

42. Dubuquoy $\mathrm{C}$, Robichon $\mathrm{C}$, Lasnier $\mathrm{F}$, et al. Distinct regulation of adiponutrin/PNPLA3 gene expression by the transcription factors ChREBP and SREBP1c in mouse and human hepatocytes. $J$ Hepatol 2011;55:145-53.

43. Reaven G. Wanted!: a standardized measurement of plasma insulin concentration. Arterioscler Thromb Vasc Biol 2011;31:954-5.

44. Robins SJ, Lyass A, Zachariah JP, et al. Insulin resistance and the relationship of a dyslipidemia to coronary heart disease: the Framingham Heart Study. Arterioscler Thromb Vasc Biol 2011;31:1208-14.

45. Williams AL, Hoofnagle JH. Ratio of serum aspartate to alanine aminotransferase in chronic hepatitis. Relationship to cirrhosis. Gastroenterology 1988;95:734-9.

46. Angulo P, Hui JM, Marchesini G, et al. The NAFLD fibrosis score: a noninvasive system that identifies liver fibrosis in patients with NAFLD. Hepatology 2007;45:846-54.

47. McPherson S, Stewart SF, Henderson E, et al. Simple non-invasive fibrosis scoring systems can reliably exclude advanced fibrosis in patients with non-alcoholic fatty liver disease. Gut 2010;59:1265-9.

48. Okanoue T, Umemura A, Yasui K, et al. Nonalcoholic fatty liver disease and nonalcoholic steatohepatitis in Japan. J Gastroenterol Hepatol 2011;26(Suppl 1):153-62.

49. Joseph AE, Saverymuttu SH, al-Sam S, et al. Comparison of liver histology with ultrasonography in assessing diffuse parenchymal liver disease. Clin Radiol 1991;43:26-31.

50. Wong VW, Chu WC, Wong GL, et al. Prevalence of non-alcoholic fatty liver disease and advanced fibrosis in Hong Kong Chinese: a population study using proton-magnetic resonance spectroscopy and transient elastography. Gut 2012;61:409-15.

51. Shepard CW, Finelli L, Alter MJ. Global epidemiology of hepatitis C virus infection. Lancet Infect Dis 2005;5:558-67.

52. Hanafiah KM, Groeger J, Flaxman AD, et al. Global epidemiology of hepatitis $C$ virus infection: new estimates of age-specific antibody to hepatitis $\mathrm{C}$ virus seroprevalence. Hepatology. Published Online First 21 Nov 2012. doi:10.1002/hep.26141

53. Pattullo V, Douglas MW, George J. Organelle dysfunction in hepatitis $C$ virus-associated steatosis: anything to learn from nonalcoholic steatohepatitis? Expert Rev Gastroenterol Hepatol 2011;5:265-77.

54. Kumagi T, Heathcote EJ. Primary biliary cirrhosis. Orphanet J Rare Dis 2008;3:1.

55. Strassburg CP. Autoimmune hepatitis. Best Pract Res Clin Gastroenterol 2010;24:667-82.

56. Oh RC, Hustead TR. Causes and evaluation of mildly elevated liver transaminase levels. Am Fam Physician 2011;84:1003-8. 